Citation: Irk, D., Mersin, M. A., "Cubic B-spline Quasi-interpolation for Numerical Solution of Regularized Long Wave Equation". Journal of Engineering Technology and Applied Sciences 3 (3) 2018 : 173-187.

\title{
CUBIC B-SPLINE QUASI-INTERPOLATION METHOD FOR REGULARIZED LONG WAVE EQUATION
}

\author{
Dursun Irk $^{\mathrm{a}^{*}}$, Mehmet Ali Mersin \\ $a^{*}$ Department of Mathematics-Computer, Eskişehir Osmangazi University, Eskişehir, Turkey \\ dirk@ogu.edu.tr \\ ${ }^{b}$ Department of Informatics, Aksaray University Aksaray, Turkey \\ mehmetalimersin@gmail.com
}

\begin{abstract}
In this study, we present a numerical method to solve the Regularized Long Wave (RLW) equation, based on cubic B-spline quasi-interpolation for the space integration and Crank-Nicolson method for the time integration. The method is tested on the problems of propagation of a solitary wave and interaction of two solitary waves. The three conservation quantities of the motion are calculated to determine the conservation properties of the proposed algorithm.
\end{abstract}

Keywords: Finite element method, B-spline, solitary wave, regularized long wave equation, Crank Nicolson

\section{Introduction}

Various phenomena in disciplines could be described by nonlinear partial differential equations (NPDEs). Numerical solution of NPDEs is very important due to just limited classes of these equation are solved analitically. One of the nonlinear evolution equations which we deal with is the Regularized Long Wave (RLW) equation. This equation was originally introduced to describe the behavior of the undular bore by Peregrine [1] who developed the first numerical method of the RLW equation using the finite difference method. Benjamin et al. [2] showed the similarity of wave solutions of the RLW equation to the wave solutions of the more widely known Korteweg-de Vries (KdV) equation. RLW equation has been solved by various numerical method including 
finite difference method [3-5], collocation method [7-12], Galerkin method [13-22], and Quadrature method [23,24].

In this paper, Crank-Nicolson method for time integration, and quasi cubic B-spline functions for space integration are used to obtain numerical solution of the RLW equation. In the test problems section, error norms and conservation quantities are calculated for the accuracy of the solution. This study is a part of the master thesis of Mersin [25]. In this thesis the numerical solution of some partial differential equations including RLW, EW, MRLW and MEW equations were solved by using quasi spline interpolation.

\section{Governing equation}

We consider the following RLW equation

$$
u_{t}+u_{x}+\varepsilon u u_{x}-\mu u_{x x t}=0
$$

with the boundary conditions

$$
u(a, t)=u(b, t)=0, t>0
$$

and the initial condition

$$
u(x, 0)=f(x)
$$

We denoted the space of univariate splines of degree $d$ which has $C^{d-1}$ property by $S_{d}\left(X_{N}\right)$ on the uniform partition

$$
X_{N}=x_{i}=a+i h, i=0, \ldots, N
$$

with the meshlength $h=\frac{b-a}{N}$, where $b=x_{N}$. Let the B-spline basis of $S_{d}\left(X_{N}\right)$ be $\left\{B_{j}, j \in J\right\}$ with $J=\{1,2, \ldots, N+d\}$, which can be computed by the de Boor-Cox formula [26]. Using the Boor-Cox formula, $B_{j}$ is obtained as

$$
B_{j}(x)=\frac{1}{6 h^{3}} \begin{cases}\left(x-x_{j}\right)^{3} & , x \in\left[x_{j}, x_{j+1}\right) \\ \left(x-x_{j}\right)^{2}\left(x_{j+2}-x\right)+ & , x \in\left[x_{j+1}, x_{j+2}\right) \\ \left(x-x_{j}\right)\left(x_{j+3}-x\right)\left(x-x_{j+1}\right)+ & \\ \left(x_{j+4}-x\right)\left(x-x_{j+1}\right)^{2} & \\ \left(x-x_{j}\right)\left(x_{j+3}-x\right)^{2}+ & , x \in\left[x_{j+2}, x_{j+3}\right) \\ \left(x-x_{j+1}\right)\left(x_{j+3}-x\right)\left(x_{j+4}-x\right)+ & , x \in\left[x_{j+3}, x_{j+4}\right) \\ \left(x_{j+4}-x\right)^{2}\left(x-x_{j+2}\right) & \text { else } \\ \left(x_{j+4}-x\right)^{3} & \end{cases}
$$

for $j \in J$. Univariate B-spline quasi-interpolants can be defined as operators of the form 


$$
Q_{d} f=\sum_{j \in I} \mu_{j} B_{j}
$$

[27-29]. For the cubic B-spline quasi interpolation

$$
Q_{3} f=\sum_{j=1}^{N+3} \mu_{j}(f) B_{j}
$$

the coefficients are listed as follows:

$$
\begin{aligned}
& \mu_{1}(f)=f_{0} \\
& \mu_{2}(f)=\frac{1}{18}\left(7 f_{0}+18 f_{1}-9 f_{2}+2 f_{3}\right), \\
& \mu_{j}(f)=\frac{1}{6}\left(-f_{j-3}+8 f_{j-2}-f_{j-1}\right), j=3, \ldots, N+1, \\
& \mu_{N+2}(f)=\frac{1}{18}\left(2 f_{N-3}+18 f_{1}-9 f_{2}+2 f_{3}\right), \\
& \mu_{N+3}(f)=f_{N} .
\end{aligned}
$$

The main advantage of quasi interpolation is having a direct construction without solving any system of linear equations.

$$
Q_{3} u=\sum_{j=1}^{N+3} \mu_{j}(u) B_{j}, \quad\left(Q_{3} u\right)^{\prime}=\sum_{j=1}^{N+3} \mu_{j}(u) B_{j}^{\prime}, \quad\left(Q_{3} u\right)^{\prime \prime}=\sum_{j=1}^{N+3} \mu_{j}(u) B_{j}^{\prime \prime}
$$

are the approximations of the first and the second derivatives of the unknown function $u$. After using these approximations

$$
\begin{aligned}
& Q u^{\prime}\left(x_{0}\right)=\frac{1}{h}\left(-\frac{11}{6} u_{0}+3 u_{1}-\frac{3}{2} u_{2}+\frac{1}{3} u_{3}\right), \\
& Q u^{\prime}\left(x_{1}\right)=\frac{1}{h}\left(-\frac{1}{3} u_{0}-\frac{1}{2} u_{1}+u_{2}-\frac{1}{6} u_{3}\right), \\
& Q u^{\prime}\left(x_{j}\right)=\frac{1}{h}\left(\frac{1}{12} u_{j-2}-\frac{2}{3} u_{j-1}+\frac{2}{3} u_{j+1}-\frac{1}{12} u_{j+2}\right), j=2, \ldots, N-2 \\
& Q u^{\prime}\left(x_{N-1}\right)=\frac{1}{h}\left(\frac{1}{6} u_{N-3}-u_{N-2}-\frac{1}{2} u_{N-1}+\frac{1}{3} u_{N}\right), \\
& Q u^{\prime}\left(x_{N}\right)=\frac{1}{h}\left(-\frac{1}{3} u_{N-3}+\frac{3}{2} u_{N-2}-3 u_{N-1}+\frac{11}{6} u_{N}\right)
\end{aligned}
$$

are obtained for the first derivation and,

$$
\begin{aligned}
& Q u^{\prime \prime}\left(x_{0}\right)=\frac{1}{h^{2}}\left(2 u_{0}-5 u_{1}+4 u_{2}-u_{3}\right), \\
& Q u^{\prime \prime}\left(x_{1}\right)=\frac{1}{h^{2}}\left(u_{0}-2 u_{1}+u_{2}\right), \\
& Q u^{\prime \prime}\left(x_{j}\right)=\frac{1}{h^{2}}\left(-\frac{1}{6} u_{j-2}+\frac{5}{3} u_{j-1}-3 u_{j}+\frac{5}{3} u_{j+1}-\frac{1}{6} u_{j+2}\right), j=2, \ldots, N-2 \\
& Q u^{\prime \prime}\left(x_{N-1}\right)=\frac{1}{h^{2}}\left(u_{N-2}-2 u_{N-1}+u_{N}\right) \\
& Q u^{\prime \prime}\left(x_{N}\right)=\frac{1}{h^{2}}\left(-u_{N-3}+4 u_{N-2}-5 u_{N-1}+2 u_{N}\right)
\end{aligned}
$$

are obtained for the second derivation [29]. 


\section{Numerical scheme}

Applying Crank Nicolson method to the equation

$$
u_{t}+u_{x}+\varepsilon u u_{x}-\mu u_{x x t}=0
$$

we have

$$
\frac{u^{n+1}-u^{n}}{\Delta t}+\frac{u_{x}^{n+1}+u_{x}^{n}}{2}+\varepsilon \frac{\left(u u_{x}\right)^{n+1}+\left(u u_{x}\right)^{n}}{2}-\mu \frac{u_{x x}^{n+1}-u_{x x}^{n}}{\Delta t}=0 .
$$

Linearizing the nonlinear term $\left(u u_{x}\right)^{n+1}$ by using following approximation [30]:

$$
\left(u u_{x}\right)^{n+1} \approx u^{n} u_{x}^{n+1}+u_{x}^{n} u^{n+1}-u^{n} u_{x}^{n}
$$

Eq. (9) can be written as

$$
\left(1+\varepsilon \frac{\Delta t}{2} u_{x}^{n}\right) u^{n+1}+\frac{\Delta t}{2}\left(1+\varepsilon\left(u^{n}\right)\right) u_{x}^{n+1}-\mu u_{x x}^{n+1}=u^{n}-\frac{\Delta t}{2} u_{x}^{n}-\mu u_{x x}^{n} .
$$

After using quasi spline approximations for the first and the second derivatives (7-8), the Eq. (11) is written as

$$
\begin{aligned}
& U_{0}^{n+1}\left[1+\varepsilon \frac{\Delta t}{2}\left(U_{x}\right)_{0}^{n}-\frac{11}{6 h} \frac{\Delta t}{2}\left(1+\varepsilon U_{0}^{n}\right)-\mu \frac{2}{h^{2}}\right]+ \\
& U_{1}^{n+1}\left[\frac{3}{h} \frac{\Delta t}{2}\left(1+\varepsilon U_{0}^{n}\right)+\mu \frac{5}{h^{2}}\right]+ \\
& U_{2}^{n+1}\left[-\frac{3}{2 h} \frac{\Delta t}{2}\left(1+\varepsilon U_{0}^{n}\right)-\mu \frac{4}{h^{2}}\right]+ \\
& U_{3}^{n+1}\left[\frac{1}{3 h} \frac{\Delta t}{2}\left(1+\varepsilon U_{0}^{n}\right)+\mu \frac{1}{h^{2}}\right]=U_{0}^{n}-\frac{\Delta t}{2}\left(U_{x}\right)_{0}^{n}-\mu\left(U_{x x}\right)_{0}^{n} \\
& U_{0}^{n+1}\left[-\frac{1}{3 h} \frac{\Delta t}{2}\left(1+\varepsilon U_{1}^{n}\right)-\mu \frac{1}{h^{2}}\right]+ \\
& U_{1}^{n+1}\left[1-\frac{1}{2 h} \frac{\Delta t}{2}\left(1+\varepsilon U_{1}^{n}\right)+\mu \frac{2}{h^{2}}\right]+ \\
& U_{2}^{n+1}\left[\frac{1}{h} \frac{\Delta t}{2}\left(1+\varepsilon U_{1}^{n}\right)-\mu \frac{1}{h^{2}}\right]+ \\
& U_{3}^{n+1}\left[-\frac{1}{6 h} \frac{\Delta t}{2}\left(1+\varepsilon U_{1}^{n}\right)\right]=U_{1}^{n}-\frac{\Delta t}{2}\left(U_{x}\right)_{1}^{n}+-\mu\left(U_{x x}\right)_{1}^{n}
\end{aligned}
$$




$$
\begin{gathered}
U_{m-2}^{n+1}\left[\frac{1}{12 h} \frac{\Delta t}{2}\left(1+\varepsilon U_{m}^{n}\right)+\mu \frac{1}{6 h^{2}}\right]+ \\
U_{m-1}^{n+1}\left[-\frac{2}{3 h} \frac{\Delta t}{2}\left(1+\varepsilon U_{m}^{n}\right)-\mu \frac{5}{3 h^{2}}\right]+ \\
U_{m}^{n+1}\left[1+\varepsilon \frac{\Delta t}{2}\left(U_{x}\right)_{m}^{n}+\mu \frac{3}{h^{2}}\right]+ \\
U_{m+1}^{n+1}\left[\frac{2}{3 h} \frac{\Delta t}{2}\left(1+\varepsilon U_{m}^{n}\right)-\mu \frac{5}{3 h^{2}}\right]+ \\
\quad U_{m+2}^{n+1}\left[-\frac{1}{12 h} \frac{\Delta t}{2}\left(1+\varepsilon U_{m}^{n}\right)+\mu \frac{1}{6 h^{2}}\right]=U_{m}^{n}-\frac{\Delta t}{2}\left(U_{x}\right)_{m}^{n}-\mu\left(U_{x x}\right)_{m}^{n} \\
U_{N-3}^{n+1}\left[\frac{1}{6 h} \frac{\Delta t}{2}\left(1+\varepsilon U_{N-1}^{n}\right)\right]+ \\
U_{N-2}^{n+1}\left[-\frac{1}{h} \frac{\Delta t}{2}\left(1+\varepsilon U_{N-1}^{n}\right)-\mu \frac{1}{h^{2}}\right]+ \\
U_{N-1}^{n+1}\left[1+\varepsilon \frac{\Delta t}{2}\left(U_{x}\right)_{N-1}^{n}-\frac{1}{2 h} \frac{\Delta t}{2}\left(1+\varepsilon U_{N-1}^{n}\right)+\mu \frac{2}{h^{2}}\right]+ \\
U_{N}^{n+1}\left[\frac{1}{3 h} \frac{\Delta t}{2}\left(1+\varepsilon U_{N-1}^{n}\right)-\mu \frac{1}{h^{2}}\right]=U_{N-1}^{n}-\frac{\Delta t}{2}\left(U_{x}\right)_{N-1}^{n}-\mu\left(U_{x x}\right)_{N-1}^{n} \\
U_{N-3}^{n+1}\left[-\frac{1}{3 h} \frac{\Delta t}{2}\left(1+\varepsilon U_{N}^{n}\right)+\mu \frac{1}{h^{2}}\right]+ \\
U_{N-2}^{n+1}\left[\frac{3}{2 h} \frac{\Delta t}{2}\left(1+\varepsilon U_{N}^{n}\right)-\mu \frac{4}{h^{2}}\right]+ \\
U_{N-1}^{n+1}\left[-\frac{3}{h} \frac{\Delta t}{2}\left(1+\varepsilon U_{N}^{n}\right)+\mu \frac{5}{h^{2}}\right]+ \\
U_{N}^{n+1}\left[1+\varepsilon \frac{\Delta t}{2}\left(U_{x}\right)_{N}^{n}+\frac{11}{6 h} \frac{\Delta t}{2}\left(1+\varepsilon U_{N}^{n}\right)-\mu \frac{2}{h^{2}}\right]=U_{N}^{n}-\frac{\Delta t}{2}\left(U_{x}\right)_{N}^{n}-\mu\left(U_{x x}\right)_{N}^{n}
\end{gathered}
$$

where $m=2, \ldots, N-2$. The system (12) contains $N+1$ unknowns and $N+1$ equations. The first and the last equations are deleted to apply the boundary conditions to the system. Then our system turns to $N+1$ unknown and $N-1$ equations.

After the first and the last equations are deleted and the boundary conditions $U(a, t)=U(b, t)=$ 0 are implemented, then the system (12) is transformed into

$$
\begin{aligned}
& U_{1}^{n+1}\left[1+\varepsilon \frac{\Delta t}{2}\left(U_{x}\right)_{1}^{n}-\frac{1}{2 h} \frac{\Delta t}{2}\left(1+\varepsilon U_{1}^{n}\right)+\mu \frac{2}{h^{2}}\right]+ \\
& U_{2}^{n+1}\left[\frac{1}{h} \frac{\Delta t}{2}\left(1+\varepsilon U_{1}^{n}\right)-\mu \frac{1}{h^{2}}\right]+ \\
& U_{3}^{n+1}\left[-\frac{1}{6 h} \frac{\Delta t}{2}\left(1+\varepsilon U_{1}^{n}\right)\right]=U_{1}^{n}-\frac{\Delta t}{2}\left(U_{x}\right)_{1}^{n}-\mu\left(U_{x x}\right)_{1}^{n}
\end{aligned}
$$




$$
\begin{aligned}
& U_{1}^{n+1}\left[-\frac{2}{3 h} \frac{\Delta t}{2}\left(1+\varepsilon U_{2}^{n}\right)-\mu \frac{5}{3 h^{2}}\right]+ \\
& U_{2}^{n+1}\left[1+\varepsilon \frac{\Delta t}{2}\left(U_{x}\right)_{2}^{n}+\mu \frac{3}{h^{2}}\right]+ \\
& U_{3}^{n+1}\left[\frac{2}{3 h} \frac{\Delta t}{2}\left(1+\varepsilon U_{2}^{n}\right)-\mu \frac{5}{3 h^{2}}\right]+ \\
& U_{4}^{n+1}\left[-\frac{1}{12 h} \frac{\Delta t}{2}\left(1+\varepsilon U_{2}^{n}\right)+\mu \frac{1}{6 h^{2}}\right]=U_{2}^{n}-\frac{\Delta t}{2}\left(U_{x}\right)_{2}^{n}-\mu\left(U_{x x}\right)_{2}^{n} \\
& U_{m-2}^{n+1}\left[\frac{1}{12 h} \frac{\Delta t}{2}\left(1+\varepsilon U_{m}^{n}\right)+\mu \frac{1}{6 h^{2}}\right]+ \\
& U_{m-1}^{n+1}\left[-\frac{2}{3 h} \frac{\Delta t}{2}\left(1+\varepsilon U_{m}^{n}\right)-\mu \frac{5}{3 h^{2}}\right]+ \\
& U_{m}^{n+1}\left[1+\varepsilon \frac{\Delta t}{2}\left(U_{x}\right)_{m}^{n}+\mu \frac{3}{h^{2}}\right]+ \\
& U_{m+1}^{n+1}\left[\frac{2}{3 h} \frac{\Delta t}{2}\left(1+\varepsilon U_{m}^{n}\right)-\mu \frac{5}{3 h^{2}}\right]+ \\
& U_{m+2}^{n+1}\left[-\frac{1}{12 h} \frac{\Delta t}{2}\left(1+\varepsilon U_{m}^{n}\right)+\mu \frac{1}{6 h^{2}}\right]=U_{m}^{n}-\frac{\Delta t}{2}\left(U_{x}\right)_{m}^{n}-\mu\left(U_{x x}\right)_{m}^{n} \\
& U_{N-4}^{n+1}\left[\frac{1}{12 h} \frac{\Delta t}{2}\left(1+\varepsilon U_{N-2}^{n}\right)+\mu \frac{1}{6 h^{2}}\right]+ \\
& U_{N-3}^{n+1}\left[-\frac{2}{3 h} \frac{\Delta t}{2}\left(1+\varepsilon U_{N-2}^{n}\right)-\mu \frac{5}{3 h^{2}}\right]+ \\
& U_{N-2}^{n+1}\left[1+\varepsilon \frac{\Delta t}{2}\left(U_{x}\right)_{N-2}^{n}+\mu \frac{3}{h^{2}}\right]+ \\
& U_{N-1}^{n+1}\left[\frac{2}{3 h} \frac{\Delta t}{2}\left(1+\varepsilon U_{N-2}^{n}\right)-\mu \frac{5}{3 h^{2}}\right]=U_{N-2}^{n}-\frac{\Delta t}{2}\left(U_{x}\right)_{N-2}^{n}-\mu\left(U_{x x}\right)_{N-2}^{n}
\end{aligned}
$$

$$
\begin{aligned}
& U_{N-3}^{n+1}\left[\frac{1}{6 h} \frac{\Delta t}{2}\left(1+\varepsilon U_{N-1}^{n}\right)\right]+ \\
& U_{N-2}^{n+1}\left[-\frac{1}{h} \frac{\Delta t}{2}\left(1+\varepsilon U_{N-1}^{n}\right)-\mu \frac{1}{h^{2}}\right]+ \\
& U_{N-1}^{n+1}\left[1+\varepsilon \frac{\Delta t}{2}\left(U_{x}\right)_{N-1}^{n}-\frac{1}{2 h} \frac{\Delta t}{2}\left(1+\varepsilon U_{N-1}^{n}\right)+\mu \frac{2}{h^{2}}\right]=U_{N-1}^{n}-\frac{\Delta t}{2}\left(U_{x}\right)_{N-1}^{n}-\mu\left(U_{x x}\right)_{N-1}^{n}
\end{aligned}
$$

where $m=3, \ldots, N-3$. It's clearly seen that the equation system consist of $N-1$ equations and $N-1$ unknowns where $U_{1}^{n+1}, U_{2}^{n+1}, \cdots, U_{N-1}^{n+1}$ are unknowns. Initial unknowns $U_{0}^{0}, U_{1}^{0}, \cdots, U_{N}^{0}$ are calculating by using initial condition $U\left(x_{m}, 0\right)=f\left(x_{m}\right) m=0,1, \ldots, N$ to solve system (13) iteratively. The local truncation error for the Eq. (13) is computed as $\frac{h^{2}}{2}\left(U_{x x x x}\right)_{m}^{n}+\frac{k^{2}}{2}\left[\mu\left(U_{x x}\right)_{m}^{n}\left(U_{x t}\right)_{m}^{n}-\mu \varepsilon^{2} \mu U_{m}^{n}\left(\left(U_{x}\right)_{m}^{n}\right)^{2}\left(U_{x x}\right)_{m}^{n}+\varepsilon \mu U_{m}^{n}\left(U_{x t}\right)_{m}^{n}\left(U_{x x}\right)_{m}^{n}\right.$ $\left.+\varepsilon \mu^{2}\left(U_{x}\right)_{m}^{n}\left(U_{x x}\right)_{m}^{n}\left(U_{x x t}\right)_{m}^{n}+\mu\left(U_{x x t}\right)_{m}^{n}\left(U_{x}\right)_{m}^{n}-\mu^{2}\left(U_{x x}\right)_{m}^{n}\left(U_{x x t t}\right)_{m}^{n}-\varepsilon \mu\left(\left(U_{x}\right)_{m}^{n}\right)^{2}\left(U_{x x}\right)_{m}^{n}\right]+\cdots$ by neglecting the terms of high order. Since the local truncation error vanishes as the time and space steps become smaller, the one step difference equation (13) is consistent with RLW equation. 


\section{The test problems}

\subsection{Motion of single solitary wave}

The exact solution of the RLW equation is given by

$$
u(x, t)=3 \operatorname{csech}^{2}\left(k\left[x-\widetilde{x_{0}}-(1+\varepsilon c) t\right]\right)
$$

which describes a single bell-shape solitary wave of amplitude $3 c$, travelling with velocity $v=1+c$ in the positive $x$-direction over the space interval $[a, b]$. The initial condition for single solitary wave solution of the RLW equation can be written as

$$
u(x, 0)=3 \operatorname{csech}^{2}\left(k\left[x-\widetilde{x_{0}}\right]\right)
$$

where $k=\sqrt{\frac{\varepsilon c}{4 \mu v}}$. The RLW equation possess three conservation constants,

$$
C_{1}=\int_{-\infty}^{\infty} u d x, \quad C_{2}=\int_{-\infty}^{\infty}\left(u^{2}+\mu\left(u_{x}\right)^{2}\right) d x, \quad C_{3}=\int_{-\infty}^{\infty}\left(u^{3}+3 u^{2}\right) d x
$$

corresponding to mass, momentum and energy, respectively [31]. Exact values of these conservation constants can be calculated by Maple as:

$$
C_{1}=\frac{6 c}{k}, \quad C_{2}=\frac{12 c^{2}}{k}+\frac{48 k c^{2} \mu}{5}, \quad C_{3}=\frac{36 c^{2}}{5 k}(4 c+5) \text {. }
$$

To compare the analytical and the numerical solutions, error norm $L_{\infty}=\max _{m}\left|U_{m}-u\left(x_{m}, t\right)\right|$ is used, where $u\left(x_{m}, t\right)$ is corresponding to the exact solution on $\left(x_{m}, t_{n}\right)$ and $U_{m}$ is corresponding to the approximate solution on $\left(x_{m}, t_{n}\right)$.

Initial solution (15) and position of solitary waves at time $t=20$ over the space interval $-40 \leq x \leq 60$ are shown at the Figure 1 for $c=0.1, \tilde{x}_{0}=0$ and parameters $\varepsilon=\mu=1$.

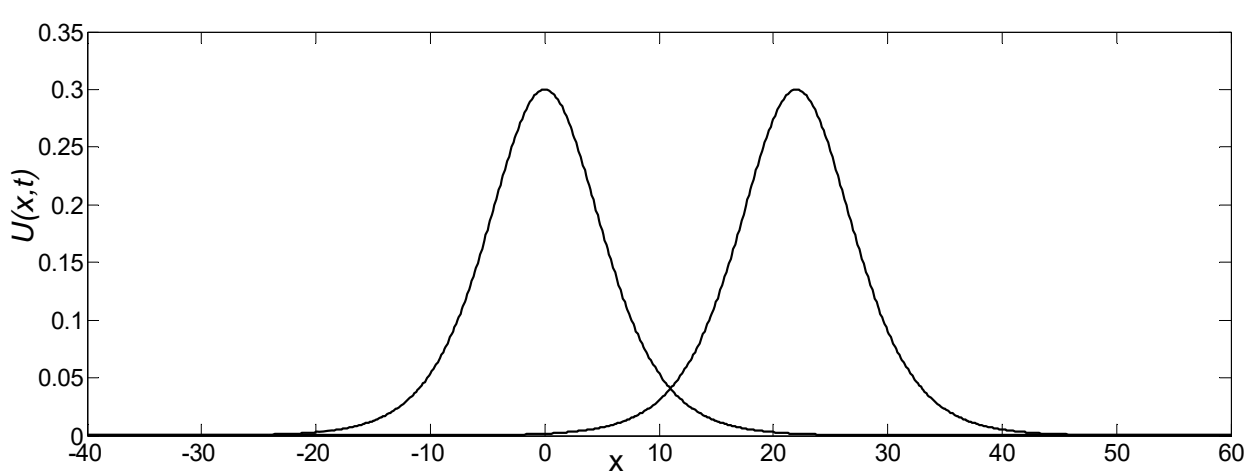

Figure 1. Solitary waves at $\boldsymbol{t}=\mathbf{0}$ and $\boldsymbol{t}=\mathbf{2 0}$ for $\boldsymbol{c}=\mathbf{0 . 1}$. 
On the first test problem parameters are chosen as space step $h=0.125$, time step $\Delta t=0.1$ and amplitude $3 c=0.3$. Program was run up to time $t=20$ and the conservation quantities $C_{1}, C_{2}$, $C_{3}$ and $L_{\infty}$ error norm are given at the Table 1 in different times. Its easy to see that the results are acceptable and in good aggrement with the exact solution according to the the error norms $L_{\infty}$ and conservation quantitites.

Table 1. Conservation quantities and the error norms

\begin{tabular}{|l|l|c|c|c|} 
for $\boldsymbol{h}=\mathbf{0 . 1 2 5}, \boldsymbol{\Delta t}=\mathbf{0 . 1}, \boldsymbol{c}=\mathbf{0 . 1}$ and $-\mathbf{4 0} \leq \boldsymbol{x} \leq \mathbf{6 0}$. \\
\hline Time & \multicolumn{1}{|c|}{$\boldsymbol{L}_{\infty} \mathbf{x} \mathbf{1 0}^{\mathbf{5}}$} & $\boldsymbol{C}_{\mathbf{1}}$ & $\boldsymbol{C}_{\mathbf{2}}$ & $\boldsymbol{C}_{\mathbf{3}}$ \\
\hline 0 & 0 & 3.9799267 & 0.8104625 & 2.5790074 \\
\hline 4 & 2.12 & 3.9799300 & 0.8104625 & 2.5790074 \\
\hline 8 & 4.26 & 3.9799267 & 0.8104625 & 2.5790074 \\
\hline 12 & 6.22 & 3.9799257 & 0.8104625 & 2.5790074 \\
\hline 16 & 8.00 & 3.9799171 & 0.8104625 & 2.5790074 \\
\hline 20 & 9.62 & 3.9798828 & 0.8104625 & 2.5790074 \\
\hline Exact & 0 & 3.9799497 & 0.8104625 & 2.5790074 \\
\hline
\end{tabular}

The absolute value of the difference between the numerical solution and the analytical solution at $t=20$ is plotted in the Figure 2. It is clear to see that the maximum error is occured in the middle of the space interval.

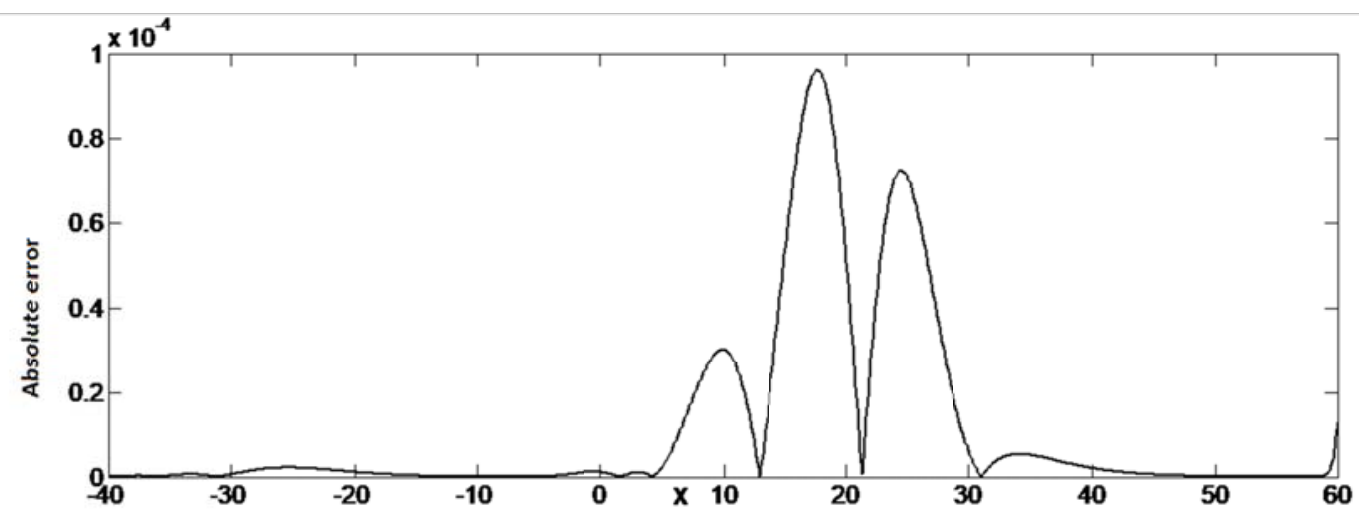

Figure 2. Absolute Error $=\mid$ Analytical Solution - Numerical Solution $\mid$ for $h=0.125, \Delta t=0.1, c=0.1$ and $-40 \leq x \leq 60$ at the time $t=20$.

The solitary wave problem is investigated by changing the amplitude of the solitary waves. Therefore, initial solution (15) and position of solitary waves at time $t=20$ over the space interval $40 \leq x \leq 60$ are shown at the Figure 3 for $c=0.03, \tilde{x}_{0}=0$ and parameters $\varepsilon=\mu=1$. 


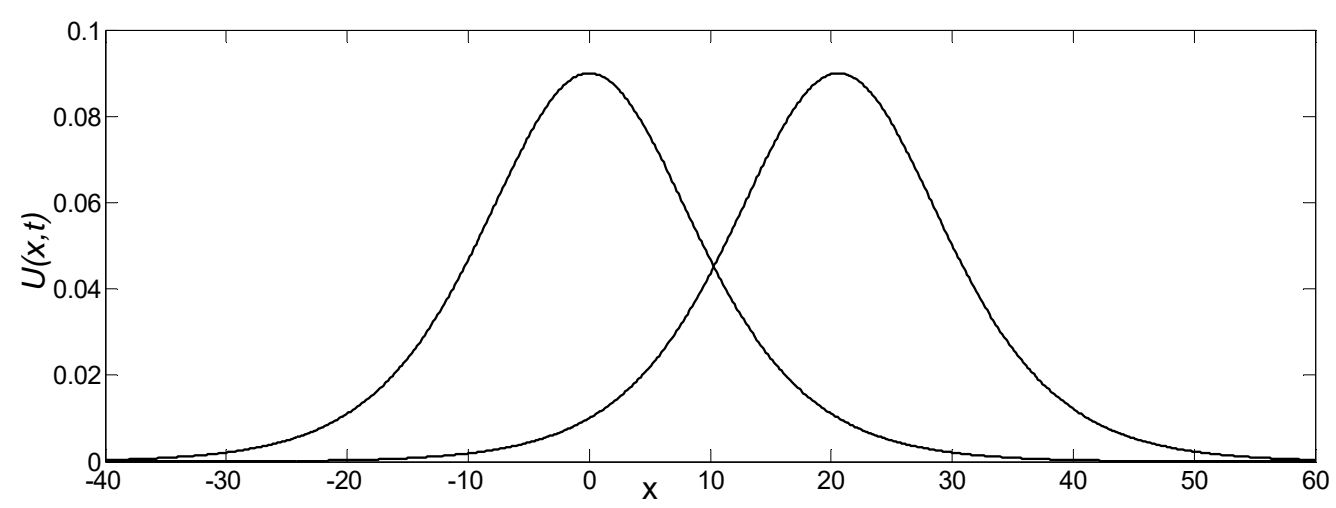

Figure 3. Solitary waves at $\boldsymbol{t}=\mathbf{0}$ and $\boldsymbol{t}=\mathbf{2 0}$ for $\boldsymbol{c}=\mathbf{0 . 0 3}$.

On this problem, parameters are choosen as space step $h=0.125$, time step $\Delta t=0.1$ and amplitude $3 c=0.09$. The program was run up to time $t=20$ and the conservation quantities $C_{1}, C_{2}, C_{3}$ and error norm $L_{\infty}$ are given at the Table 2 by different times. According to the Table 2 its easy to see the absolute error has increased according to $c=0.1$.

Table 2. Conservation quantities and the error norm for $h=0.125, \Delta t=0.1, c=0.03$ and $-40 \leq x \leq 60$.

\begin{tabular}{|l|l|c|c|c|}
\hline Time & \multicolumn{1}{|c|}{$\boldsymbol{L}_{\infty} \mathbf{X 1 0}^{\mathbf{4}}$} & $\boldsymbol{C}_{\mathbf{1}}$ & $\boldsymbol{C}_{\mathbf{2}}$ & $\boldsymbol{C}_{\mathbf{3}}$ \\
\hline 0 & 0 & 2.10704672 & 0.12730126 & 0.38880465 \\
\hline 4 & 2.30 & 2.10709771 & 0.12730112 & 0.38880407 \\
\hline 8 & 2.21 & 2.10689617 & 0.12730112 & 0.38880405 \\
\hline 12 & 2.12 & 2.10654963 & 0.12730111 & 0.38880397 \\
\hline 16 & 2.14 & 2.10592816 & 0.12730109 & 0.38880365 \\
\hline 20 & 4.32 & 2.10461363 & 0.12730104 & 0.38880235 \\
\hline
\end{tabular}

Figure 4 shows the absolute value of the difference between the analytical and numerical solutions of the program. The program was run up to time $t=20$ with $h=0.125, \Delta t=0.1, c=0.03$ over the space interval $-40 \leq x \leq 60$. The maximum error can be seen at the end of the space interval. It means that there is a problem with the boundary conditions. The reason for the error seen at the end of the space domain is due to the space interval of the wave is not chosen close enough to the zero. 


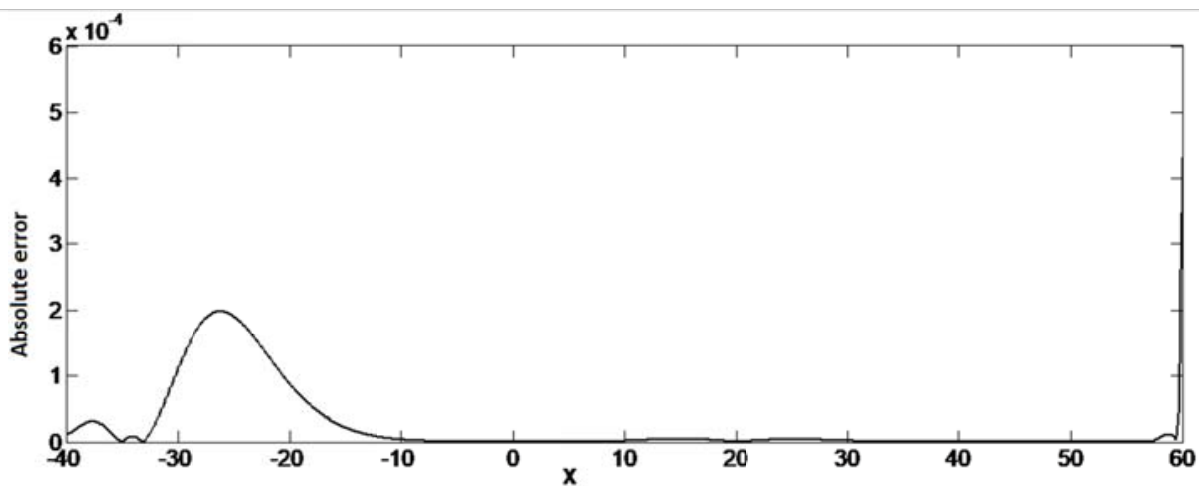

Figure 4. Absolute Error $=\mid$ Analytical Solution - Numerical Solution $\mid$ $0.125, \Delta t=0.1, c=0.03$ and $-40 \leq x \leq 60$ at the time $t=20$.

In order to reduce the error, the program was run again by extending the space interval from $-40 \leq x \leq 60$ to $-80 \leq x \leq 120$. The obtained results are given in the Table 3 . It can be seen from the table that the error is decreased when the larger space interval $-80 \leq x \leq 120$ is used.

Table 3. Conservation quantities and the error norms for $h=0.125, \Delta t=0.1, c=0.03$ and $-80 \leq x \leq 120$.

\begin{tabular}{|l|l|l|l|l|}
\hline Time & \multicolumn{1}{|c|}{$\boldsymbol{L}_{\infty} \mathbf{X 1 0}^{\mathbf{6}}$} & $\boldsymbol{C}_{\mathbf{1}}$ & $\boldsymbol{C}_{\mathbf{2}}$ & $\boldsymbol{C}_{\mathbf{3}}$ \\
\hline 0 & 0 & 2.10940502 & 0.12730172 & 0.38880599 \\
\hline 4 & 0.88 & 2.10940516 & 0.12730172 & 0.38880599 \\
\hline 8 & 1.79 & 2.10940511 & 0.12730172 & 0.38880599 \\
\hline 12 & 2.71 & 2.10940507 & 0.12730172 & 0.38880599 \\
\hline 16 & 3.65 & 2.10940508 & 0.12730172 & 0.38880599 \\
\hline 20 & 4.57 & 2.10940502 & 0.12730172 & 0.38880599 \\
\hline Exact & & 2.10940750 & 0.12730172 & 0.38880599 \\
\hline
\end{tabular}

The Figure 5 shows the absolute value of the difference between the analytical and numerical solutions at time $t=20$ over the interval of $-80 \leq x \leq 120$ and it is seen that the error is decreased and the maximum error is also occurred in the middle of the interval. As a result, the selection of boundary conditions is very important in the solitary wave test problems.

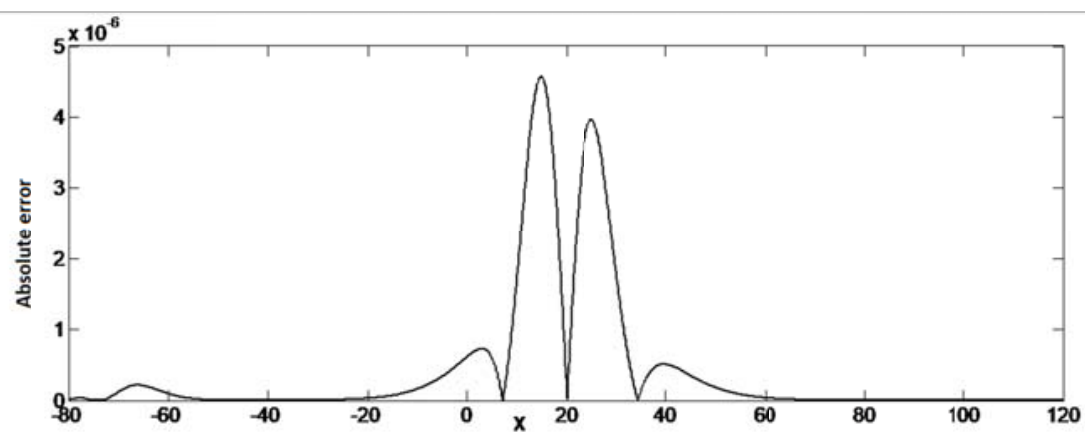

Figure 5. Absolute Error $=\mid$ Analytical Solution - Numerical Solution $\mid$ for $h=0.125, \Delta t=0.1, c=0.03$ and $-80 \leq x \leq 120$ at the time $t=20$. 
Table 4 displays a comparison of the values of the invariants and error norms obtained by the present method with those obtained by other methods. The results of the proposed method in good agreement with previous studies results.

Table 4. Conservation quantities and the error norms for $h=0.125, \Delta t=0.1, c=0.1$ and $-40 \leq x \leq 60$ at time $t=20$.

\begin{tabular}{|c|c|c|c|c|}
\hline Method & $L_{\infty} \times 10^{5}$ & $C_{1}$ & $C_{2}$ & $C_{3}$ \\
\hline Present & 9.62 & 3.9798828 & 0.8104625 & 2.5790074 \\
\hline [13] & 8.6 & 3.97988 & 0.810465 & 2.57901 \\
\hline [18] & 175.5 & \begin{tabular}{|l|}
3.98203 \\
\end{tabular} & 0.808650 & 2.57302 \\
\hline [19] & 156.6 & 3.96160 & 0.804185 & 2.55829 \\
\hline [21] & 19.8 & 3.98206 & 0.811164 & 2.58133 \\
\hline [22] & 7.34 & 3.9798879 & 0.8104622 & 2.5790063 \\
\hline Exact & 7 & 3.979949 & 0.8104625 & 2.5790074 \\
\hline
\end{tabular}

\subsection{Interaction of two solitary waves}

The collision problem of two solitary waves has the following initial condition

$$
u(x, 0)=3 c_{1} \operatorname{sech}^{2}\left(k_{1}\left[x-\widetilde{x_{1}}\right]\right)+3 c_{2} \operatorname{sech}^{2}\left(k_{2}\left[x-\widetilde{x_{2}}\right]\right)
$$

where $k_{i}=\sqrt{\frac{\varepsilon c_{i}}{4 \mu\left(1+\varepsilon c_{i}\right)}}, i=1,2$. In (18), the solitary waves have $3 c_{1}$ and $3 c_{2}$ amplitudes, and each peak points of them are located at $\widetilde{x_{1}}$ and $\widetilde{x_{2}}$, respectively over the problem domain $[a, b]$. If the parameters are choosen as $c_{1}>c_{2}$ and $\widetilde{x_{2}}>\widetilde{x_{1}}$ in the initial solution (18), the bigger wave which has bigger amplitude will stand on the left. Hence, if the parameters are choosen properly, the bigger wave will reach and pass the smaller wave as its faster than the smaller wave. So the collision will be occured.

Exact values of the conservation constants for this problem can be calculated by Maple as

$$
\begin{aligned}
& C_{1}=6\left(\frac{c_{1}}{k_{1}}+\frac{c_{2}}{k_{2}}\right), \\
& C_{2}=12\left(\frac{c_{1}^{2}}{k_{1}}+\frac{c_{2}^{2}}{k_{2}}\right)+\frac{48}{5} \mu\left(k_{1} c_{1}^{2}+k_{2} c_{2}^{2}\right), \\
& C_{3}=\frac{36 c_{1}^{2}}{5 k_{1}}\left(4 c_{1}+5\right)+\frac{36 c_{2}^{2}}{5 k_{2}}\left(4 c_{2}+5\right) .
\end{aligned}
$$

When the parameters are choosen as $\widetilde{x_{1}}=20, \widetilde{x_{2}}=65, c_{1}=2 / 3$ and $c_{2}=0.1$, the initial condition of RLW equation for the interecation of two solitary waves test problem is

$$
u(x, 0)=2 \operatorname{sech}^{2}\left(\frac{\sqrt{10}}{10}[x-20]\right)+0.3 \operatorname{sech}^{2}\left(\frac{1}{\sqrt{44}}[x-65]\right) .
$$

The program was run up to time $t=150$ with the parameters $h=\Delta t=0.1$ over the space interval $[0,300]$. The solitary waves' at times $t=0, t=65$ and $t=150$ are plotted in the Figure 6. It is observed from the Figure 6 that the time of the collision is around $t=65$ and the 
solitary waves retain their shape after the collision.

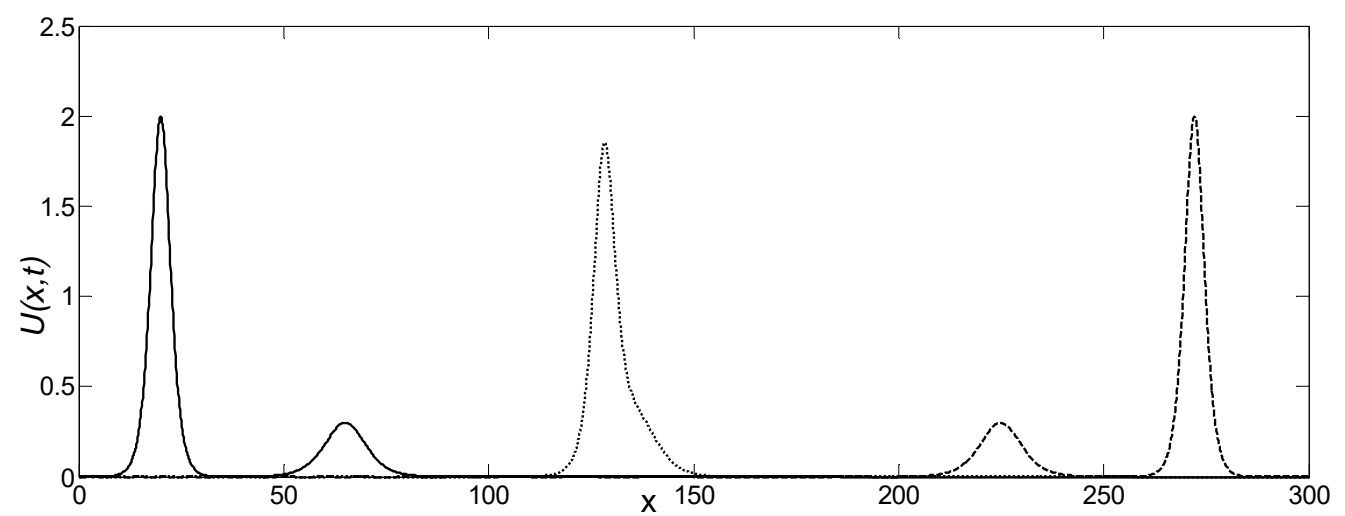

Figure 6. Collision of two solitary waves at times $t=0, t=65, t=150$.

The numerical values of the conservation constants are given in Table 5 for various times.

Table 5. Conservation quantities for the collision of two solitary waves.

\begin{tabular}{|l|c|c|c|}
\hline Time & $\boldsymbol{C}_{\mathbf{1}}$ & $\boldsymbol{C}_{\mathbf{2}}$ & $\boldsymbol{C}_{\mathbf{3}}$ \\
\hline 0 & 16.62901975 & 19.02523346 & 80.16048477 \\
\hline 30 & 16.62903736 & 19.02519099 & 80.16021819 \\
\hline 60 & 16.62903725 & 19.02299037 & 80.14604732 \\
\hline 90 & 16.62903719 & 19.02393162 & 80.15214565 \\
\hline 120 & 16.62903854 & 19.02519877 & 80.16026810 \\
\hline 150 & 16.62903891 & 19.02520778 & 80.16032521 \\
\hline
\end{tabular}

Absolute errors for conservation constants are given in the Figure 7. In this figure, the largest error occurs for $C_{3}$, then for $C_{2}$, and the smallest error for $C_{1}$. It shows that the absolute error of the conservation constants is increasing and then decreasing between $t=50$ and $t=100$ because of the collision. 


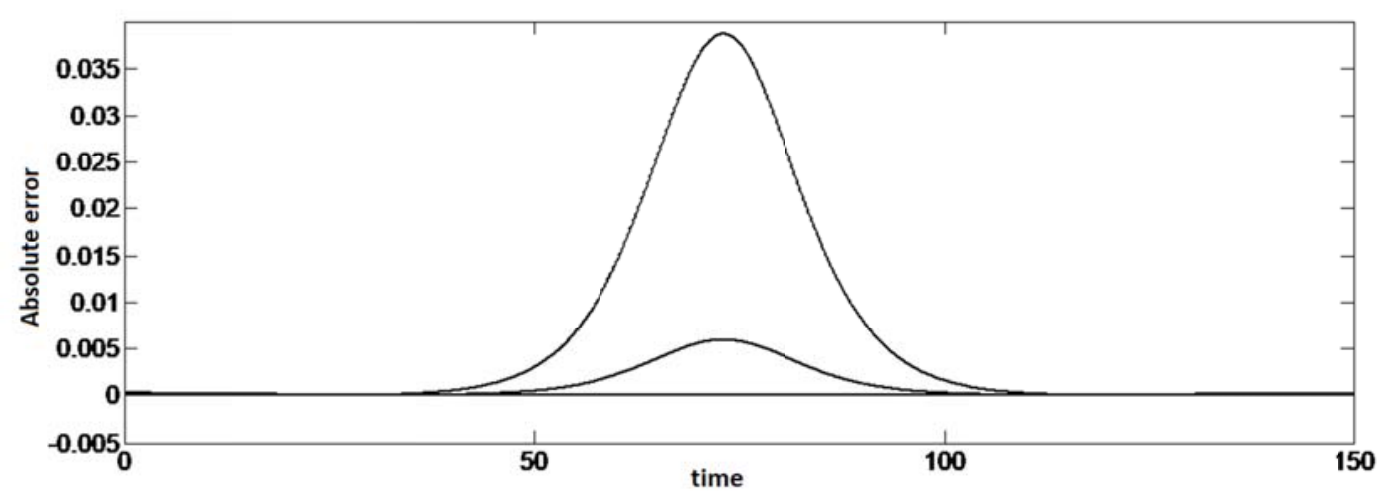

Figure 7. Absolute errors for conservative quantities.

\section{Conclusion}

In this study, quasi cubic B-splines for space discretization and Cranck Nicolson method for time discretization are proposed for numerical solution of the RLW equation. The advantage of the proposed method is the unknown function can be directly calculated by approaching the derivatives as in the finite difference method. The proposed numerical method for the RLW equation is investigated by two test problems. After the numerical solutions are examined, it is seen that the proposed method gives good results. Consequently, quasi cubic B-spline method which is an easier method than the finite element method in the terms of ease of implementation for the numerical solution of the RLW equation.

\section{References}

[1] Peregrine, D.H., "Calculations of the development of an undular bore”, J. Fluid. Mech. 25 (2) (1966): 321-330.

[2] Benjamin, T.B., Bona, J.L., and Mahony, J.J., "Model equations for long waves in non-linear dispersive systems", Philos. Trans. R. Soc., London A 272 (1972): 47-78.

[3] Eilbeck, J.C., and McGuire, G.R. "Numerical study of RLW equation" I: numerical methods", J. Comput. Phys. 19 (1975) 43-57.

[4] Eilbeck, J.C. and McGuire, G. R., "Numerical study of the regularized long-wave equation II: interaction of solitary waves", Journal of Computational Physics 23, (1977): 63-73.

[5] Padam, C.J. and Iskandar, L. "Numerical solutions of the regularized long wave equation", Comp. Methods Appl. Mech. Eng. 20 (1979): 195-201.

[6] Irk, D., Dag, I. and Dogan, A., "Numerical integration of the RLW equation using cubic splines", Anziam Journal 47, (2005): 131-142.

[7] Raslan, K.R., "A computational method for the regularized long wave (RLW) equation", Applied Mathematics and Computation 167(2), (2005b): 1101-1118.

[8] Soliman, A. A. and Hussien, M. H., "Collocation solution for RLW equation with septic 
spline", Applied Mathematics and Computation 161, (2005):623-636.

[9] Saka, B., Dag, I. and Irk, D., "Quintic B-spline collocation method for numerical solution of the RLW equation", Anziam Journal 49, (2008b): 389-410.

[10] Saka, B., Sahin, A. and Dag, I., "B-spline collocation algorithms for numerical solution of the RLW equation", Wiley Subscription Services, Inc., A Wiley Company 27, 3, (2011): 581-607.

[11] Irk, D., "Solitary wave solutions for the regularized long-wave equation", Physics of Wave Phenomena 20, 3, (2012):174-183.

[12] Saka, B., and Dag, I. "A collocation method for the numerical solution of the RLW equation using cubic B-spline basis”, Arabian Journal for Science and Engineering. 30. 39-50, (2005).

[13] Esen, A. and Kutluay, S., "Application of a lumped Galerkin method to the regularized long wave equation", Applied Mathematics and Computation 174, (2006):833-845.

[14] Alexander, M.E. and Morris, J.LL. "Galerkin methods applied to some model equations for nonlinear dispersive waves”, J. Comput. Phys. 30 (1979): 428-451.

[15] Gardner, L.R.T. and Gardner, G.A. "Solitary wave of the regularised long wave equation", J. Comput. Phys. 91 (1990): 441-459.

[16] Gardner, L.R.T., Gardner, G.A. and Dag, I. "A B-spline finite element method for the regularized long wave equation", Commun. Numer., Methods Eng. 11 (1995): 59-68.

[17] Gardner, L.R.T. and I. Dag, I. "The boundary-forced regularised long-wave equation", Il Nuo. Cimen. 110B (12), (1995): 1487-1495.

[18] Gardner, L.R.T., Gardner, G.A. and Dogan, A. "A least squares finite element scheme for the RLW equation", Commun. Numer., Methods Eng. 12 (1996): 795-804.

[19] Dag, I. and Ozer, M.N. "Approximation of RLW equation by least square cubic B-spline finite element method", Appl. Math. Model. 25 (2001): 221-231.

[20] Dogan, A., "Numerical solution of regularized long wave equation using Petrov-Galerkin method", Communications in Numerical Methods in Engineering 17, (2001):485-494.

[21] Dogan, A., "Numerical solution of RLW equation using linear finite elements within Galerkin's method", Applied Mathematical Modelling 26(7), (2002): 771-783.

[22] Saka, B. and Dag, I., "A numerical solution of the RLW equation by Galerkin method using quartic B-splines", Communications in Numerical Methods in Engineering 24, (2008):1339-1361.

[23] Dag, I., Korkmaz A. and Saka, B., "Cosine expansion-based differential quadrature algorithm for numerical solution of the RLW equation" 26, 3, (2010): 544-560.

[24] Korkmaz, A. and Dag, I. "Numerical Simulations of Boundary-Forced RLW Equation with Cubic B-Spline-based Differential Quadrature Methods", Arabian Journal for Science and Engineering, Volume 38, Issue 5, pp (2013): 1151-1160.

[25] Mersin M.A. "B-spline Quasi-Interpolation Method for Numerical Solutions of some Partial Differential Equations", M.S., Eskişehir Osmangazi University, (2014), 80p.

[26] Farin, G., "Curves and Surfaces for CAGD", fifth ed., Morgan Kaufman, San Francisco, (2001).

[27] Sablonnière, P., "Quasi-interpolants splines sobre particiones uniforms, in: First Meeting in 
Approximation Theory of the University of Jaén, Ubeda" Prépublication IRMAR 00-38, Rennes, (June 29-July 2, 2000).

[28] Sablonnière, P., "Univariate spline quasi-interpolants and applications to numerical analysis”, Rend. Sem. Mat. Univ. Pol. Torino 63, (2005): 211-222.

[29] Zhu, C. G. and Kang, W. S., "Numerical solution of Burgers-Fisher equation by cubic B-spline quasi-interpolation", Applied Mathematics and Computation 216, (2010):2679-2686.

[30] Rubin S. G. and Graves R. A., "A Cubic Spline Approximation for Problems in Fluid Mechanics," Nasa TR R-436, Washington, DC, (1975).

[31] Olver, P.J. "Euler operators and conservation laws of the BBM equation", Math. Proc. Camb. Philos. Soc. 85 (1979): 143-159. 